\title{
Customized vs Conventional Abutments in Healing Fresh Extraction Dental Sockets on Maxillary Anterior Teeth
}

\author{
Menchini-Fabris Giovanni-Battista ${ }^{1}$, Covani Ugo ${ }^{2}$, Toti Paolo $^{3}$, Crespi Giovanni ${ }^{4}$, Rubino Luigi ${ }^{5}$, Crespi Roberto $^{6}$
}

\begin{abstract}
Aim: The aim of the present retrospective study on fresh socket implants in esthetic region was to compare customized vs standard healing abutments, evaluating alveolar bone assessment.

Materials and methods: Postextractive sockets underwent immediate dental implant placement without filling the voids between the implant surface and socket wall. Width of the alveolar ridge following implant placement with or without customized abutment mimicking the look of the extracted tooth was measured on three-dimensional radiographs before and 3 years after surgery. The statistic is performed with a level of significance of 0.05 .

Results: In a split-mouth analysis, 22 patients were enrolled with a total of 44 selected maxillary dental implants: 14 incisives, 14 canines, and 16 premolars. An implant survival rate of $100 \%$ was reported for all implants after 36 months. 3 years after the implant placement, loss in bone width was registered both for the standard group $(-2.18 \pm 0.59 \mathrm{~mm})$ and for the custom group $(-0.08 \pm 0.22 \mathrm{~mm})$, even if dimensional change of the alveolar ridge following the healing procedure appeared in the custom group to be not significant with respect to the standard group. Significant differences were found between groups irrespective of tooth type ( $p \leq 0.0001)$, and among subgroups related to the tooth type (incisor vs both canine and premolar, with $p \leq 0.0124$ ).

Conclusion: Custom procedure might help provide a seal over the surgical site to protect the coagulum and to support natural emergence profile for optimal final restorative contours, and in doing so promoting socket volume maintenance.

Keywords: Cone-beam computed tomography, Customized abutment, Dental abutment, Dental implant, Immediate dental implant loading, Immediate implant placement.

International Journal of Prosthodontics and Restorative Dentistry (2019): 10.5005/jp-journals-10019-1239
\end{abstract}

\section{INTRODUCTION}

Immediate implant placement in fresh extraction socket reduces the number of surgeries and the duration of treatment, with improvement of overall patient comfort and satisfaction. However, in the esthetic zone, the insertion of the implant immediately after extraction can lead to esthetic disharmonies regarding the risk of facial recession of the peri-implant mucosa. ${ }^{1}$

There is evidence that the occurrence of sudden and unforeseeable resorption of the coronal portion of the buccal bone plate, which tends to be very thin in the anterior maxilla, could be concomitant with facial recession. ${ }^{2,3}$

Immediate implant placement is considered a complex surgical procedure. The most important requirements are an intact facial bony plate with a thickness higher than $1 \mathrm{~mm}$, a thick biotype of gingiva, ${ }^{4}$ and the presence of at least $2 \mathrm{~mm}$ gap between the implant and the internal surface of the facial bone wall. These anatomical features provide a favorable environment which can promote new bone formation within the bone defect region between the exposed implant surface and the facial bone wall. In order to improve esthetic results for patients treated with immediate implants into fresh extraction sockets in the maxillary sites, clinical trials suggested following critical key factors: (1) atraumatic tooth removal and minimal or no flap elevation; (2) use of a bone filler material into bony gap between immediate freshsocket implant and socket walls; and (3) a socket seal device, that is screw-retained provisional restoration.

Abovementioned factors are crucial to limit the amount of dimensional changes of the ridge contour of the fresh extraction socket, and potentially to increase thickness of the peri-implant mucosa immediately coronal to the implant-abutment interface. ${ }^{5}$
1,3,6 Department of Multidisciplinary Regenerative Research, Guglielmo Marconi University, Rome, Italy; Tuscan Stomatologic Institute, San Camillo Hospital, Forte dei Marmi, Italy

${ }^{2}$ Tuscan Stomatologic Institute, San Camillo Hospital, Forte dei Marmi, Italy

4,5 Department of Multidisciplinary Regenerative Research, Guglielmo Marconi University, Rome, Italy

Corresponding Author: Menchini-Fabris Giovanni-Battista, Department of Multidisciplinary Regenerative Research, Guglielmo Marconi University, Rome, Italy, Phone: +39 3397157007, e-mail: gb.menchinifabris@ gmail.com

How to cite this article: Giovanni-Battista M-F, Ugo C, Paolo T, et al. Customized vs Conventional Abutments in Healing Fresh Extraction Dental Sockets on Maxillary Anterior Teeth. Int J Prosthodont Restor Dent 2019;9(3):82-87.

Source of support: Nil

Conflict of interest: None

The placement of biomaterials into the void of fresh extraction socket provides advantages in the preservation of the existing osseous and gingival architecture. Nevertheless, several clinical and prosthetic challenges, for example, demanding tooth removal and placement of a bone graft material into such a big gap to the level of the free gingival margin, might lead to damage the implant-supported temporary crown, which may be critical for the esthetic outcome. .,7 $^{6}$

In those cases when immediate provisionalization and loading might not be considered to be appropriate, the use of a custom 
healing abutment contoured and shaped like an existing fresh extraction socket was proposed. ${ }^{8,9}$ Some clinicians might find the fabrication of a custom healing abutment too complicated and unsafe probably due to working with acrylic or composite in a bleeding field. Moreover, a filling by bone substitutes embodied into the socket could interfere with healing.

The aim of the present retrospective study was to compare custom vs standard healing abutments placed on fresh socket implants in the esthetic sites treated without using filler material between implant surface and the socket wall.

\section{Materials and Methods}

\section{Patient Selection}

Twenty-two patients requiring extractions of teeth from premolar to premolar regions of the maxilla due to root fractures, caries, endodontic lesions, or periodontal disease were selected for the present retrospective study, among consecutive patients treated at Tuscan Stomatologic Institute between February 2014 and April 2015, and were followed up till 3 years after surgery for period 2014-2018 at the Complex Operating Unit of Maxillo-Facial Surgery of the University of Pisa. The following inclusion criteria were adopted for each patient:

- Need of extraction of maxillary anterior teeth (from premolar to premolar) due to root fractures, decays, endodontic lesions, or periodontal disease;

- Patients in good general health (without chronic systemic diseases):

- Presence of four bony walls of the alveolus;

- Presence of at least $4 \mathrm{~mm}$ of bone beyond the root apex;

- Rehabilitation with dental implants, placed in the fresh extraction sockets;

- Both customized and standard healing abutment;

- Cone-beam computed tomography (CBCT) scans before tooth extraction and after surgery (2-3 years).Patients were excluded if items of the following information were shown in the medical record:

- Report of dehiscence or fenestration in the residual bony walls after tooth extraction;

- Report of acute infection at implant site and healing;

- Heavy smoking habit (>10 cigarettes a day);

- Alcohol or drug abuse, and oral parafunctional habits (bruxism).

All the interventions were performed by a single oral surgeon and a single prosthetic specialist. The study was conducted according to the principles embodied in the Helsinki declaration of 1975, revised in 2000, for biomedical research involving human subjects. The authors analyzed preexisting and no identifiable data of patients, who were all informed about the nature of data treatment, and all patients signed a written informed consent form for the retrospective data analysis. Present study did not require approval by a review board due to its retrospective data analysis in which commercially available materials were used, and standard operating procedures for routine clinical practice (procedures which are very well-established within 2/3 decades, such as fixed prostheses on osseointegrated dental implants) were applied.

Clinicians adhered to standard treatment guidelines according to which it was possible to routinely prescribe CBCT for diagnosed and surgical planning (preoperative or before tooth extractions). An additional CBCT scan was required to determine the appropriate surgical approach in the event of additional clinical needs (postoperative).

\section{Surgical Procedure}

1 hour prior to surgery, the patients received $1 \mathrm{~g}$ amoxicillin (Zimox, Pfizer Italia, Latina, Italy) and $1 \mathrm{~g}$ two times a day for a week after surgical procedure. Surgery was performed under local anesthesia (optocaine, Molteni Dental, Scandicci, Firenze, Italy) $20 \mathrm{mg} / \mathrm{mL}$ with adrenaline 1:80,000. Maxillary teeth (from premolar to incisive region) were extracted with Magnetic Mallet $^{10}$ (Magnetic Mallet, Meta-Ergonomica, Turbigo, Milano, Italy) while clinician focused on maintaining the integrity of the socket, and avoiding buccal and palatal flaps (Fig. 1). After tooth extraction, a periodontal probe (Hu-Friedy PGF-GFS, Hu-Friedy, Chicago, IL, USA) was used to verify the integrity of the four walls of the fresh sockets. Implants were positioned immediately after tooth extraction. No regenerative procedures were performed, no material was used for filling voids between implant surface and alveolus walls. Implant sites were prepared with a standard drill at least $4 \mathrm{~mm}$ beyond the root apex following the palatal bony walls as guide.

Lack of bone-to-implant contact at facial aspect of the anterior extraction socket could be caused by insertion of dental implant placed tilted to the palatal side depending on width and depth of the gap. All implants were titanium plasma sprayed with a rough surface, a body with a progressive thread design (Outlink, Sweden and Martina, Padova, Italy), a short smooth collar of $0.5 \mathrm{~mm}$ and external hexagon as implant-abutment junctions. The implant platform was placed at the level of the facial alveolar bone crest. The quality of alveolar bone was classified prior to implant placement as quality three, according to Lekholm and Zarb classification, then assessed during dental implant surgery. ${ }^{11}$

Into the implants of a single maxillary hemiarch either custom healing abutment (group I) or standard abutments (group II) were screwed (Fig. 1). In group I, transfer copings were connected to the external hex of the implants and secured with screws. Impression was obtained with silicone material and a custom impression tray.

The core of custom abutment was processed by subtractive milling in polyether ether ketone (PEEK) material (BioHPP, Bredent Medical GmbH \& Co.KG, Weissenhorner, Germany) and screwed on the implant. The custom abutment was fabricated immediately after surgery (within 4 hours after implant placement) by the CAD/ CAM milling method entirely out of PEEK, and similar to the shape of crown emergence profile of the extracted tooth. It should mimic the natural tooth being replaced, with the same size and shape as the natural extracted tooth in order to sustain the socket soft tissues.

The standard abutments were commercially available.

After the abutment connection, 5 to 0 silk sutures were placed.

\section{Prosthetic Procedure}

After 3 months, new impression coping was connected to the implant, and direct registration of the emergence form was captured. The master impression was then made according to the restorative treatment plan. The custom impression coping and the master impression were sent to the laboratory. Proper final abutments/temporary restorations (cement- or screw-retained) were fabricated. 6 months after implant insertion, final prosthetic restoration was performed (Fig. 1).

\section{Radiographic Examination}

The CBCT scans were performed with a device dedicated to threedimensional dental imaging (Gendex GXCB-500; Gendex Dental 

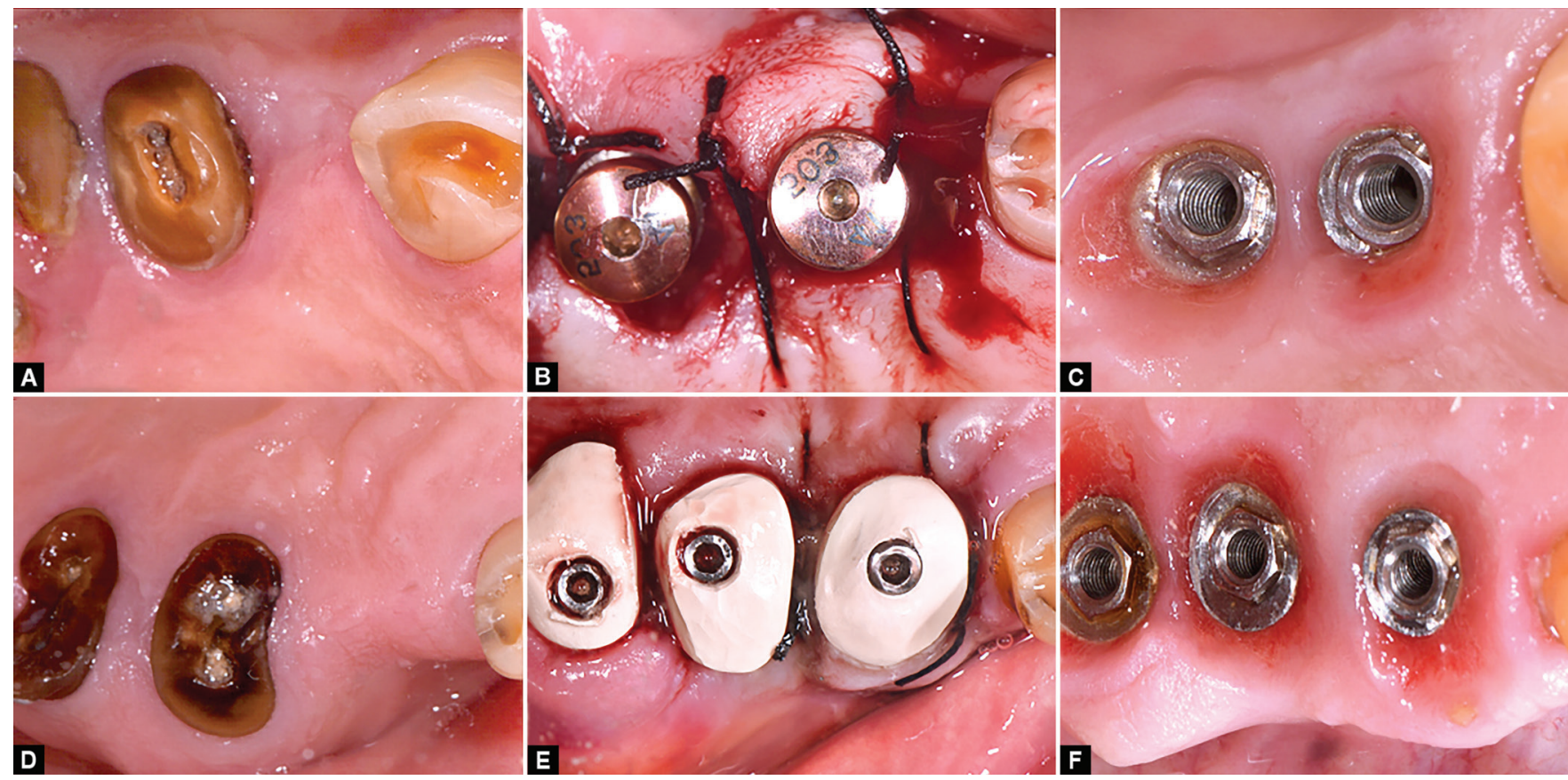

Figs $1 \mathrm{~A}$ to F: Standard abutment: (A) Clinical occlusal view of regions to be restored; (B) Standard healing abutments screwed onto the implant; (C) Clinical occlusal view of implant restored regions 3 months after implant placement; custom abutment; (D) Clinical occlusal view of regions to be restored; (E) Custom abutments screwed onto the implant; (F) Clinical occlusal view of implant restored regions 3 months after implant placement
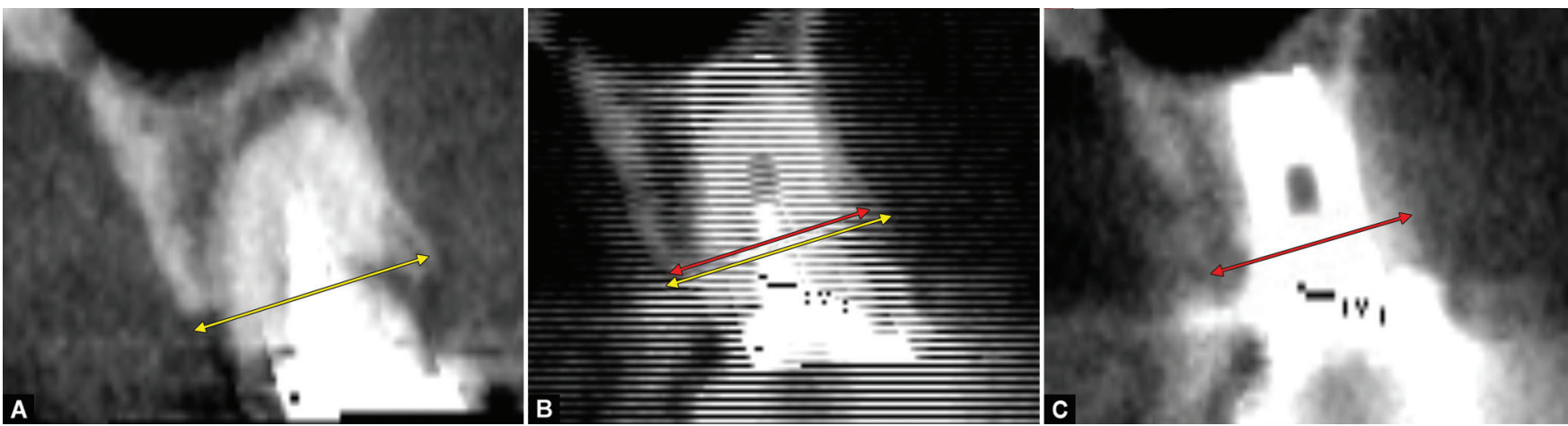

Figs $2 \mathrm{~A}$ to $\mathrm{C}$ : Cone-beam computed tomography (CBCT) preoperative view. Measurement of width in $\mathrm{CBCT}$ scans (yellow preoperative and red postoperative); (A) The preoperative CBCT scan was taken before tooth extraction; (B) CBCT scans fused (preoperative and postoperative); (C) The postoperative CBCT within 3 years after implant placement

Systems 1910 North Penn Road Hatfield, PA 19440). The following setting was the same for every acquisition: $120 \mathrm{kV}, 30.89 \mathrm{~mA}$ second, isotropic voxel size of $0.2 \mathrm{~mm}$ and $87.2 \mathrm{~mm}$ diameter of field of view. Free i-CAT Vision ${ }^{\mathrm{TM}}$ viewing software was used to analyze images. Before measurements, the two dentascans were fused as per Crespi et al. ${ }^{12}$

A CBCT cross-sectional image was extrapolated and analyzed at longitudinal coronal portion. Measurement of the alveolar width was performed perpendicular to the direction of the implant, that is, the distance between the most prominent points from the palatal to the buccal side, at $1 \mathrm{~mm}$ apical to the most coronal point (Fig. 2). The loss in bone width was the difference between preoperative (before tooth extraction) and postoperative (3 years after implant placement) measurements.

\section{Statistical Analysis}

Statistical analyses were performed with a statistical software (Statistics Toolbox, MatLab 7.11; The MathWorks). Normal distributions of the groups and subgroups were not confirmed. The two custom and standard groups were dependent on a splitmouth retrospective analysis with one treated site per group: each pair-wise comparison was performed by Wilcoxon signed rank test. Tooth position and time (preoperative vs postoperative) showed matched data; for unmatched data Kruskal-Wallis test, then post hoc Wilcoxon rank sum test was applied. Variables are described as mean \pm standard deviation (rounded to the nearest decimal). The significance was set at $p$ value $<0.05$.

\section{RESULTS}

A total of 22 patients ( 14 women and 8 men), with an age of $49.2 \pm 6.9$ years (range from 39-64 years), were selected for the present analysis. Four patients were smokers (two women and two men). A total of 56 maxillary teeth in incisor, canine, and premolar regions were extracted. Out of all 44 enrolled dental implants, 32 had a diameter of $4.2 \mathrm{~mm}$ and a length of $13 \mathrm{~mm}$; 
eight implants had a diameter of $3.75 \mathrm{~mm}$ and a length of $13 \mathrm{~mm}$; whereas four implants had a diameter of $4.2 \mathrm{~mm}$ and a length of $10 \mathrm{~mm}$. No report of postoperative bony wall sockets defect was registered in the patients' case sheet. The split-mouth retrospective analysis led to a sample size reduction from the 56 enrolled to 44 selected dental implants. In group I, 22 immediate implants were placed in fresh sockets and as many custom abutments were screwed. In group II, conventional abutments were screwed onto implants simultaneously placed in each of the two maxillary sites in the same patient.

\section{Surgical and Prosthetic Procedures}

After a 36-month follow-up period, the implant survival rate was $100 \%$ for both groups. There was no patient withdrawal in either group. Minor swelling of gingival mucosa was observed 1 day after surgery, but neither mucositis nor flap dehiscence with suppuration was encountered.

The final zirconia ceramic restorations were cemented or screwed 4 months after surgery. Neither pain nor prosthesis mobility was registered within 36 months of follow-up. Final restorative contour followed that of the natural tooth so allowing for a predictable emergence profile and a gingival embrasure form.

\section{Radiographic Evaluation}

Sockets of the custom group showed neither buccal nor palatal bone reduction after 3-year follow-up. Mean bone width before extraction was $8.36 \pm 1.21 \mathrm{~mm}$ and $8.11 \pm 1.49 \mathrm{~mm}$ for groups I and II, respectively (Table 1). 3 years after implant placement, radiographic examinations showed loss of the bone width both for the custom $-(-0.08 \pm 0.22 \mathrm{~mm})$ and the normal-group $(-2.18 \pm$ $0.59 \mathrm{~mm})$. Significant differences were found between the two groups ( $p \leq 0.0001)$ and among subgroups of different tooth site (incisor, canine, and premolar, with $p \leq 0.0156$ ) regarding bone width loss at 3-year follow-up (Figs 3 and 4).

In the intragroup analysis, change in bone width appeared negligible when sites were compared (incisor, canine, and premolar) with bone changes ranging from $+0.05 \pm 0.25 \mathrm{~mm}$ to $-0.19 \pm$ $0.16 \mathrm{~mm}$ for the custom group (Kruskal-Wallis $p=0.1178$ ); whereas the standard group incisors seemed to undergo significantly (Kruskal-Wallis $p=0.0112$ ) smaller shrinkage (with a bone loss of $1.59 \pm 0.44 \mathrm{~mm}$ and $p \leq 0.0124$ ) compared with those reported for

Table 1: Buccal width (BW) at baseline (BW preoperative ) and at 3 years follow-up ( $\mathrm{BW}_{3 \text { years }}$ ) with related changes from preoperative to 3 years (BW preoperative $\rightarrow 3$ years ) for the customized abutment group (I) and for the normal abutment group (II).

\begin{tabular}{|c|c|c|c|c|c|c|c|c|}
\hline \multirow[b]{2}{*}{ Patient } & \multirow[b]{2}{*}{ Tooth } & \multicolumn{3}{|c|}{ Custom } & \multicolumn{3}{|c|}{ Standard } & \multirow{2}{*}{$\begin{array}{l}\text { Custom } \leftrightarrow \text { standard } \\
B W_{\text {preoperative } \rightarrow 3 \text { years }} \\
p \text { value }\end{array}$} \\
\hline & & $\begin{array}{l}B W_{\text {preoperative }} \\
(\mathrm{mm})\end{array}$ & $\begin{array}{l}B W_{3 \text { years }} \\
(\mathrm{mm})\end{array}$ & $\begin{array}{l}B W_{\text {preoperative } \rightarrow 3 \text { years }} \\
(\mathrm{mm})\end{array}$ & $\begin{array}{l}B W_{\text {preoperative }} \\
(\mathrm{mm})\end{array}$ & $\begin{array}{l}B W_{3 \text { years }} \\
(\mathrm{mm})\end{array}$ & $\begin{array}{l}B W_{\text {preoperative } \rightarrow 3 \text { years }} \\
(\mathrm{mm})\end{array}$ & \\
\hline 22 & Overall & $8.36 \pm 1.21$ & $8.28 \pm 1.22$ & $-0.08 \pm 0.22$ & $8.11 \pm 1.49$ & $5.92 \pm 1.06$ & $-2.18 \pm 0.59$ & $<0.0001^{\circ}$ \\
\hline 7 & Incisor (inc) & $7.58 \pm 0.76$ & $7.45 \pm 0.72$ & $-0.13 \pm 0.15$ & $6.43 \pm 0.82$ & $4.83 \pm 0.66$ & $-1.59 \pm 0.44$ & $0.0156^{\circ}$ \\
\hline 7 & Canine (can) & $8.90 \pm 1.37$ & $8.71 \pm 1.38$ & $-0.19 \pm 0.16$ & $9.31 \pm 1.00$ & $6.74 \pm 0.89$ & $-2.57 \pm 0.53$ & $0.0156^{\circ}$ \\
\hline 8 & $\begin{array}{l}\text { Premolar } \\
\text { (prem) }\end{array}$ & $8.58 \pm 1.15$ & $8.63 \pm 1.18$ & $0.05 \pm 0.25$ & $8.52 \pm 0.85$ & $6.16 \pm 0.62$ & $-2.36 \pm 0.32$ & $0.0078^{\circ}$ \\
\hline \multicolumn{9}{|c|}{ Intragroup analysis } \\
\hline $7 \leftrightarrow 7$ & inc $\leftrightarrow$ can & & & $0.5210^{*}$ & & & $0.0082^{*}$ & \\
\hline $7 \leftrightarrow 8$ & inc $\leftrightarrow$ prem & & & $0.1582^{*}$ & & & $0.0124^{*}$ & \\
\hline $7 \leftrightarrow 8$ & can $\leftrightarrow$ p rem & & & $0.0665^{*}$ & & & $0.3207^{*}$ & \\
\hline
\end{tabular}

'Wilcoxon sign-rank test for matched samples; *Wilcoxon rank-sum test for independent samples

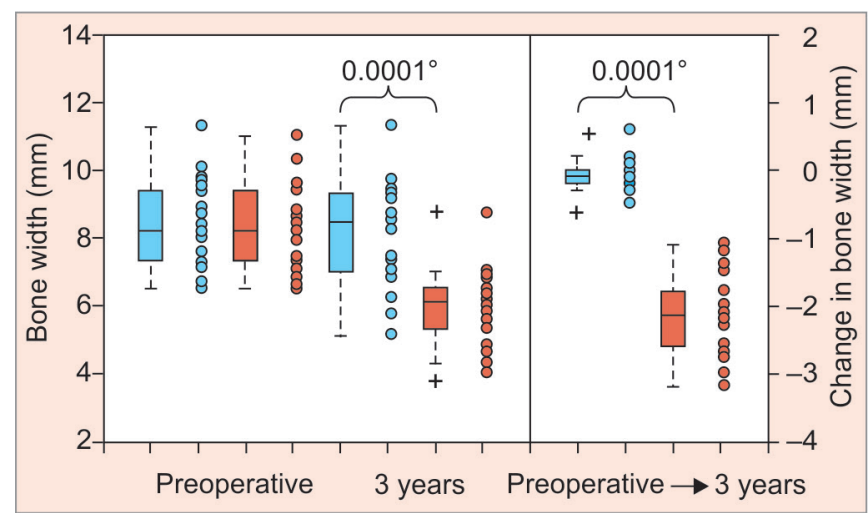

Fig. 3: Box and scatter plots for preoperative (preop) and postoperative (3 years) bone width measurements and loss in bone width (preop $\rightarrow 3$ years) expressed in $\mathrm{mm}$ for customized-group (in blue); and for standard-group (in red). ${ }^{\circ}$ Wilcoxon sign-rank test for matched samples

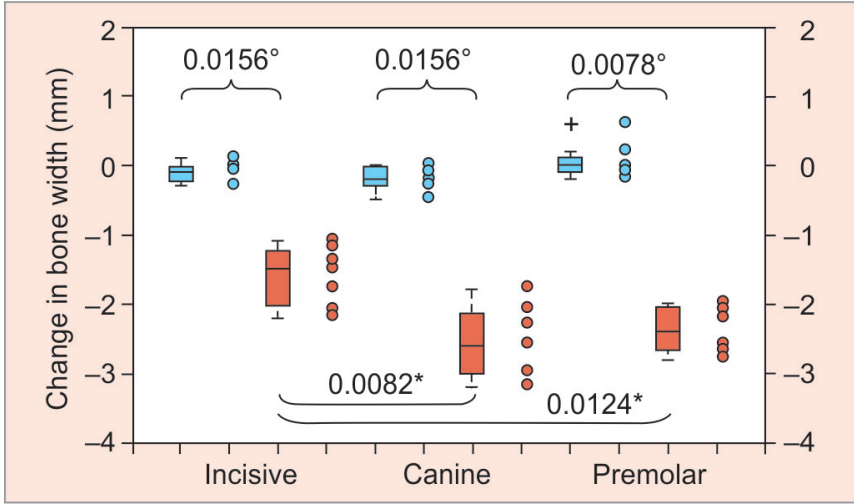

Fig. 4: Box plots for loss in bone width (preop $\rightarrow 3$ years) expressed in $\mathrm{mm}$ for customized-group (in blue); and for standard-group (in red). Implant site (incisor, canine and premolar) have been investigated by Kruskal-Wallis test (KW) and post hoc pairwise comparison tests: 'Wilcoxon sign-rank test for matched samples; and *Wilcoxon rank-sum test for independent samples. Custom group KW: SS: 174.6, df: 2, MS: $87.3, \chi^{2}: 4.2776, p$ value: 0.1178 , standard group KW: $S S: 378.3$, df: 2 , MS: $189.1, \chi^{2}: 8.9864, p$ value: 0.011 
the others $(2.57 \pm 0.53 \mathrm{~mm}$ and $2.36 \pm 0.32$, in canine and premolar site, respectively, Table 1 and Fig. 4).

\section{Discussion}

A complex root extraction is generally accompanied by soft and hard alterations of the alveolar ridge due to releasing incisions with coronally positioned injured flaps; on the contrary, during root extraction alveolus integrity is mandatory to achieve an optimal soft tissue management for immediate implant therapy in the sockets. The surgery damages soft tissue and lengthens the healing period. For group II, it was reported that alveolar bone at coronal part of the implant undergoes shrinkage and remodeling leading to a worsening of morphology of the socket. These results may be explained by the fact that stock healing abutments presented an unsuitable emergence profile for fresh socket. In addition, about closing the gap between standard abutment and gingival contour, different authors suggested to make a mimicking of the in situ customized gingival emergence profile, ${ }^{13}$ or to advocate flap elevation, mobilization and closure around the abutment for the purpose of retaining particulate grafting.

In group I, custom healing abutments not only supported gingival tissue but also prevented buccal bone collapse during the osseointegration phase. In doing so, the integrity of the alveoli during the restorative phase was maintained. The custom healing abutment constituted a mechanical and defensive barrier for the blood clot (which was stabilized) and for the socket (which was sealed), in that it prevented a premature loading of the implant during healing.

The fabrication of the emergence profile of the implant abutment and final restoration with purpose to mimic the natural dentition, included the ability to maintain support for soft tissue through an osseointegration phase protected by an accurate custom socket seal over the immediate implant. The present clinical and radiographic study suggested that the fabrication of a custom abutment is efficacious in maintaining preoperative crestal contour of the socket without the use of biomaterials (membranes and grafts). As shown by clinical and radiographic images (Figs 1 and 2) a full maintenance of both the facial bone wall dimension and the gingival tissue contour could be correct.

A recent study described the use of a precast polymethyl methacrylate shell to fabricate a custom healing abutment. ${ }^{9}$ As precast shell more closely approximated the anatomy of the cervical root area of the removed tooth, it could be modified to support the subgingival contour. In the present protocol, anatomic gingival contour was virtually designed by duplicating the anatomical shape of the existing tooth, rather than mimicking it by using conventional laboratory techniques.

Finelle and Lee reported a technique combining the use of a three-dimensional printed surgical template for guided implant surgery and CAD/CAM technology to optimize tissues healing after bone graft and sealing socket abutment. ${ }^{14}$

In the abovementioned studies, different biomaterials were used to fill the gap between implant surfaces and sockets walls. Furthermore, the sealing socket abutment technique allowed to separate the grafted area from the growth of soft tissue without any demanding surgery.

Tarnow and Chu showed clinical and histological evidence, thus demonstrating that an intact buccal wall is important for the healing process of immediate implant irrespective of gap dimension, flap closure, and biomaterials (graft and membrane). ${ }^{15}$ The histologic specimens showed that bone can quickly reach the surface when the socket is allowed to heal by secondary intention, as occurs routinely with dental extractions. Gingival tissue appeared to migrate to the clot, not until the blood clot is replaced with granulation tissue. When extraction procedure damages cortical wall, the cancellous bone of the socket is lacking in its shield. The spongy bone has high surface area and it is contiguous with the marrow compartment which is filled with marrow-derived mesenchymal progenitor cells and with endothelial population needed for angiogenesis. Around implants, trabecular and cortical bone had different remodeling levels; trabecular bone contained a rich vasculature supports and its healing usually proceeded at a faster rate than that of cortical one. ${ }^{16}$

However, what is normally seen during secondary intention healing in an extraction socket after implant installation is an accelerate formation of bone-to-implant contact before the soft tissue has the opportunity to interfere. ${ }^{15}$

As reported in the present study, an abutment with a proper emergence profile may support both the gingival and the bone tissue healing after immediate implant placement, so promoting a more predictable final restoration and a satisfactory socket volume maintenance. Custom healing abutments should be placed to preserve the anatomy and gingival esthetic present at the time of extraction, and should be viewed as the final step in the implant surgery. ${ }^{17}$

As regards the advantages of the present study, an estimated clinical preeminence of the customized immediate abutment is not yet supported by superimposition of the volumetric preoperative and postoperative data. Limitations of the present study may be its retrospective nature, and the possible presence of errors generated by the metal artifacts. Finally, the limited number of the sample may be another bias.

\section{Conclusion}

Custom procedure might help to provide a seal over the surgical site to protect blood clot and to support natural emergence profile for optimal final restorative contours, and in doing so it promotes socket volume maintenance. Present encouraging results to obtain a natural anatomy of the implant-prosthetic restoration without demanding procedures need to be further verified once larger datasets become available.

\section{References}

1. Evans CD, Chen ST. Esthetic outcomes of immediate implant placements. Clin Oral Implants Res 2008;19(1):73-80. DOI: 10.1111/j.1600-0501.2007.01413.x.

2. Hämmerle $\mathrm{CH}$, Araújo MG, Simion M, et al. Evidence-based knowledge on the biology and treatment of extraction sockets. Clin Oral Implants Res 2012;23(Suppl 5):80-82. DOI: 10.1111/j.1600-0501.2011. 02370.x.

3. Benic GI, Mokti M, Chen CJ, et al. Dimensions of buccal bone and mucosa at immediately placed implants after 7 years: a clinical and cone beam computed tomography study. Clin Oral Implants Res 2012;23(5):560-566. DOI: 10.1111/j.1600-0501.2011.02253.x.

4. Morton D, Chen ST, Martin WC, et al. Consensus statements and recommended clinical procedures regarding optimizing esthetic outcomes in implant dentistry. Int J Oral Maxillofac Implants 2014;29(Suppl):216-220. DOI: 10.11607/jomi.2013.g3.

5. Buser D, Chappuis V, Belser UC, et al. Implant placement post extraction in esthetic single tooth sites: when immediate, when early, when late? Periodontol 2000 2017;73(1):84-102. DOI: 10.1111/ prd.12170. 
6. Trimpou G, Weigl P, Krebs M, et al. Rationale for esthetic tissue preservation of a fresh extraction socket by an implant treatment concept simulating a tooth replantation. Dent Traumatol 2010;26(1):105-111. DOI: 10.1111/j.1600-9657.2009.00831.x.

7. Castelnuovo J, Sonmez AB. The autogenous immediate implant supported single-tooth restoration: a 5-year follow-up. Eur J Esthet Dent 2012;7(4):382-395.

8. Tarnow DP, Chu SJ, Salama MA, et al. Flapless postextraction socket implant placement in the esthetic zone: part 1. The effect of bone grafting and/or provisional restoration on facial-palatal ridge dimensional change - a retrospective cohort study. Int J Periodontics Restorative Dent 2014;34(3):323-331. DOI: 10.11607/prd.1821.

9. Chu SJ, Hochman MN, Tan-Chu JH, et al. A novel prosthetic device and method for guided tissue preservation of immediate postextraction socket implants. Int J Periodontics Restorative Dent 2014;34(Suppl 3):s9-s17. DOI: 10.11607/prd.1749.

10. Crespi R, Bruschi GB, Capparé $P$, et al. The utility of the electric mallet. J Craniofac Surg 2014;25(3):793-795. DOI: 10.1097/ SCS.0000000000000523.

11. Lekholm U, Zarb GA. Patient selection and preparation. In: Brånemark P-I, Zarb GA, Albrektsson T, ed. Tissue-integrated prostheses: osseointegration in clinical dentistry. Chicago: Quintessence; 1985. pp. 201-209.
12. Crespi R, Fabris GBM, Crespi G, et al. Effects of different loading protocols on the bone remodeling volume of immediate maxillary single implants: a 2- to 3-year follow-up. Int J Oral Maxillofac Implants 2019;34(4):953-962. DOI: 10.11607/jomi.6972

13. Araujo MG, Linder E, Lindhe J. Bio-Oss collagen in the buccal gap at immediate implants: a 6-month study in the dog. Clin Oral Implants Res 2011;22(1):1-8. DOI: 10.1111/j.1600-0501.2010.01920.x.

14. Finelle G, Lee SJ. Guided immediate implant placement with wound closure by computer-aided design/computer-assisted manufacture sealing socket abutment: case report. Int J Oral Maxillofac Implants 2017;32(2):e63-e67. DOI: 10.11607/jomi.4770.

15. Tarnow DT, Chu SJ. Human histologic verification of osseointegration of an immediate implant placed into a fresh extraction socket with excessive gap distance without primary flap closure, graft, or membrane: a case report. Int J Periodontics Restorative Dent 2011;31(5):515-552.

16. Franchi $M$, Fini $M$, Martini $D$, et al. Biological fixation of endosseous implants. Micron 2005;36(7-8):665-671. DOI: 10.1016/ j.micron.2005.05.010.

17. Richard Akin. A new concept in maintaining the emergence profile in immediate posterior implant placement: the anatomic harmony abutment. J Oral Maxillofac Surg 2016;74(12):2385-2392. DOI: 10.1016/j.joms.2016.06.184. 